\title{
Existence of positive skewness of velocity gradient in early transition
}

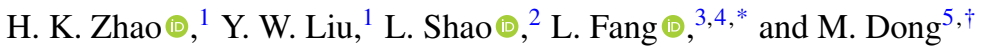 \\ ${ }^{1}$ National Key Laboratory of Science and Technology on Aero-Engine Aero-Thermodynamics, \\ School of Energy and Power Engineering, Beihang University, Beijing 100191, China \\ ${ }^{2}$ LMFA, CNRS, Ecole Centrale de Lyon-Université de Lyon, 69130 Ecully, France \\ ${ }^{3}$ Laboratory of Complex Systems, Ecole Centrale de Pékin, Beihang University, Beijing 100191, China \\ ${ }^{4}$ Beihang Hangzhou Innovation Institute Yuhang, Hangzhou 310034, China \\ ${ }^{5}$ State Key Laboratory of Nonlinear Mechanics, Institute of Mechanics, Chinese Academy of Sciences, \\ Beijing 100190, China
}

(Received 17 May 2021; accepted 13 October 2021; published 25 October 2021)

\begin{abstract}
The present study uses direct numerical simulations to calculate two different transitional flows from laminar flow to turbulence, that is, the two-scale wake flow and the two-dimensional Reyleigh-Taylor unstable flow, respectively. Both results show that the skewness of the longitudinal velocity gradient, $S_{k}$, can become positive in the early transition stage, which is beyond our expectation since the turbulent equilibrium state always implies negative values of $S_{k}$. These phenomena are explained analytically by considering only two dominant Fourier modes with harmonic relations. It is illustrated that the sign of $S_{k}$ is not only affected by the amplitudes of the perturbation velocities, but also related to their phases. We expect that the present results will be helpful for understanding the formation of turbulent equilibrium state and for constructing a new measurable criterion of the transition onset.
\end{abstract}

DOI: 10.1103/PhysRevFluids.6.104608

\section{INTRODUCTION}

Turbulence is usually regarded as a chaotic dynamic system characterized by the presence of various temporal and spacial scales. A distinguished phenomenon of turbulence is the interscale energy transfer. When energy is transferred from large to small scales, it is referred to as the forward transfer or energy cascade [1]; by contrast, the inverse energy cascade, also called energy backscatter, refers to the energy transfer from small to large scales. In physical space, the interscale energy transfer can be quantitatively represented by involving the concept of the third-order moments of the velocity gradient or velocity increment $[2,3]$. Specifically, the skewness of the longitudinal velocity gradient is defined as

$$
S_{k}:=\left\langle\left(\frac{\partial u}{\partial x}\right)^{3}\right\rangle /\left\langle\left(\frac{\partial u}{\partial x}\right)^{2}\right\rangle^{3 / 2},
$$

with $x$ being the longitudinal direction, $u$ the fluctuation of longitudinal velocity component, and \langle\rangle the ensemble averaging. In three-dimensional statistically steady and fully developed turbulence, it is already known that $S_{k}$ is a negative constant which weakly depends on Reynolds number [4-7]. The negative sign corresponds to the forward interscale energy transfer, which supports Kolmogorov's 1941 theory [1,2]. In turbulence, the change of the sign of $S_{k}$ or nonconstant

\footnotetext{
*Corresponding author: le.fang@buaa.edu.cn
}

†Corresponding author: dongming@imech.ac.cn 
value of $S_{k}$ means that the flow is not in a statistically equilibrium state, which corresponds to a self-organizing process among various turbulence scales. For example, a Gaussian random field yields null $S_{k}$; an incompressible isotropic two-dimensional turbulence also leads to null $S_{k}$ (see Refs. [8,9] for discussions); and in some typical initial conditions the energy transfer is suppressed in a very short time period [10,11], while $S_{k}$ is accordingly near zero. We also observed positive $S_{k}$ when reversing the velocities in a fully developed three-dimensional turbulence $[12,13]$, which leads to a strong nonequilibrium procedure afterwards [14-16]. Indeed, positive $S_{k}$ in real turbulence, such as flow in air compressors [17], usually indicates a signal of energy backscatter.

In a recent direct numerical simulation (DNS), we found that in the early transition stage of spatial-developing channel flow, the value of $S_{k}$ can be unexpectedly positive [9]. This fact could be surprising because intuitively we should expect $S_{k}$ to decrease monotonically from 0 (Gaussian random field) to the negative constant (turbulence), as predicted in turbulence closure theories [4]. The explanation of this phenomenon in Ref. [9] is an incomplete tentative. Moreover, we do not even know if this positive $S_{k}$ is universal or occasional. In this sense, we attempt to carry out more investigations to reveal the behavior of $S_{k}$. We will report the statistical results of two numerical configurations which respectively correspond to different scenarios of transition to turbulence to argue that the phenomenon of positive $S_{k}$ can occur in the early transition phase. Analytical approximations and modelings are then presented to better explain these phenomena. Note that the present observations only consider the skewness of streamwise velocity gradient as a characteristic quantity in early transition of anisotropic flows without involving any local isotropy assumption on velocity gradient moments.

In the following sections, we will present two different transition flows respectively: a two-scale wake in Sec. II and a two-dimensional Reyleigh-Taylor flow in Sec. III. Both cases will show positive $S_{k}$ in the early transition stage. Analytical models then will be introduced to explain this phenomenon, in Secs. II C and III C, respectively. Discussion of the underlying mechanism and the perspectives will be given in Sec. IV.

\section{TRANSITION IN A TWO-SCALE WAKE}

In order to investigate the phenomenon of positive skewness in early transition, in this section we design a numerical experiment for a two-scale wake. This case is an extreme simplification of the wake transition and can also be interpreted as the experiment of a two-scale grid-generated turbulence. Only two Fourier modes with harmonic relations are introduced as the inlet condition, which simplifies the description of the wake flow and enables the analytical approximation in the subsections.

\section{A. Simulation settings}

We consider a perfect-gas uniform flow with infinitesimal noises propagating through a semipermeable membrane panel of two-scale grids, which generates vorticities and fluctuations with different scales in the wake of the panel, leading to transition to turbulence eventually. The flow field is described in the three-dimensional Cartesian coordinate system $\left(x_{1}, x_{2}, x_{3}\right)$, with $x_{1}$ along the streamwise direction and the origin $o$ at the panel. For a selected cuboid computational domain, $[-0.4 L, 16 L] \times[0,2 L] \times[0,2 L], 1640 \times 200 \times 200$ grid points are allocated with uniform width $\Delta$ in all directions, where $L$ is the characteristic length scale of the grid panel. Periodic conditions are employed in the two transverse directions $\left(x_{2}\right.$ and $\left.x_{3}\right)$. See Fig. 1 for a sketch. The velocity field $\left(U_{1}, U_{2}, U_{3}\right)$, density $\rho$, pressure $P$ and temperature $T$ are normalized by $U_{\infty}, \rho_{\infty}, \rho_{\infty} U_{\infty}^{2}$, $T_{\infty}$, where the subscript $\infty$ denotes the dimensional oncoming mean quantities. Two dimensionless parameters are introduced, $M=U_{\infty} / a_{\infty}$ and $R e=U_{\infty} L / \nu_{\infty}$, where $a_{\infty}$ and $\nu_{\infty}$ are the sound speed and kinematic viscosity of the oncoming stream. 


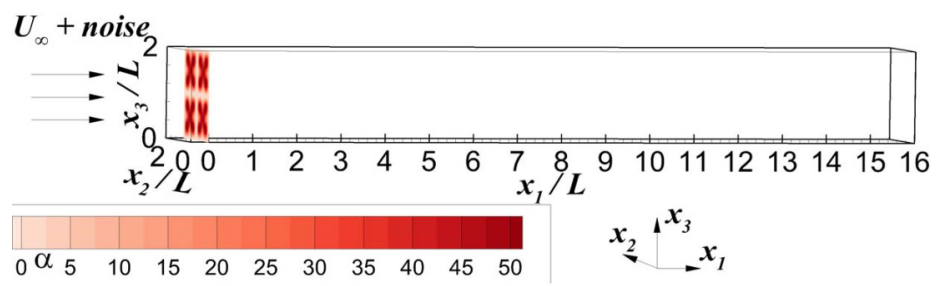

(a)

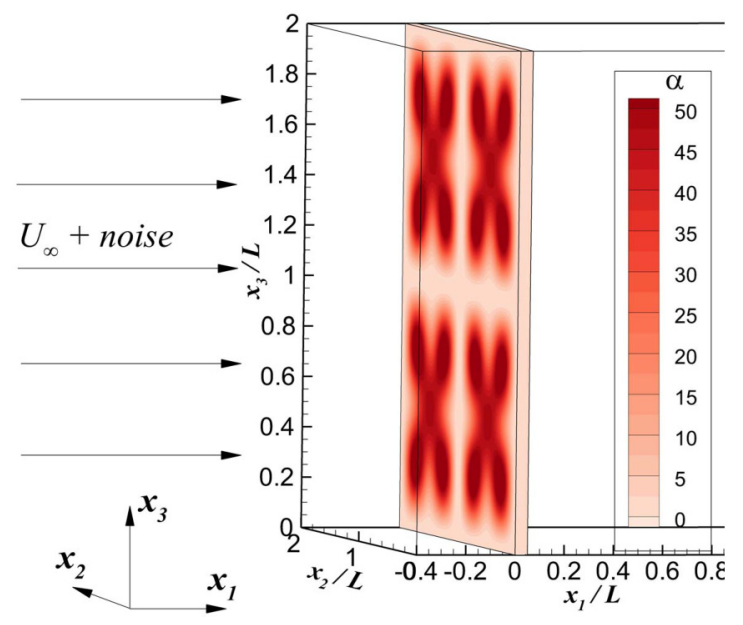

(b)

FIG. 1. Sketch of the computational domain of the two-scale wake. The membrane is highlighted. (a) Global view; (b) zoomed view near inlet.

The dimensionless governing equations for the compressible flow are

$$
\begin{gathered}
\frac{\partial \rho}{\partial t}+\frac{\partial\left(\rho U_{k}\right)}{\partial x_{k}}=0 \\
\frac{\partial\left(\rho U_{i}\right)}{\partial t}+\frac{\partial\left(\rho U_{k} U_{i}\right)}{\partial x_{k}}=-\frac{\partial P}{\partial x_{i}}+\frac{\partial \tau_{i k}}{\partial x_{k}}-\alpha \rho U_{i}, \\
\frac{\partial E}{\partial t}+\frac{\partial\left[(E+P) U_{k}\right]}{\partial x_{k}}=\frac{\partial b_{k}}{\partial x_{k}}-\frac{1}{2} \alpha \rho U_{k} U_{k},
\end{gathered}
$$

where the total energy $E$ is defined as

$$
E=\frac{1}{2} \rho U_{k} U_{k}+\frac{\rho T}{\Gamma(\Gamma-1) \mathrm{Ma}^{2}},
$$

with $\Gamma=1.4$ the specific heat capacity ratio. The stress tensor and heat flux vector are

$$
\begin{aligned}
\tau_{i j} & =\frac{\mu}{\operatorname{Re}}\left(\frac{\partial U_{i}}{\partial x_{j}}+\frac{\partial U_{j}}{\partial x_{i}}-\frac{2}{3} \delta_{i j} \frac{\partial U_{k}}{\partial x_{k}}\right), \\
b_{i} & =U_{k} \tau_{i k}+\frac{\mu}{\operatorname{Pr} \operatorname{Re}(\Gamma-1) \mathrm{Ma}^{2}} \frac{\partial T}{\partial x_{i}}
\end{aligned}
$$


respectively, where $\delta_{i j}$ is Kronecker delta, $\operatorname{Pr}=0.72$ the Prandtl number, and $\mu$ the dynamic viscosity determined by the Sutherland law

$$
\mu=T^{1.5} \frac{T_{S} / T_{\mathrm{ref}}+1}{T+T_{S} / T_{\mathrm{ref}}},
$$

with $T_{\text {ref }}=298.15 \mathrm{~K}$ and $T_{S}=110.4 \mathrm{~K}$. Additionally, the equation of the state for a perfect gas determines the relation between $P, \rho$, and $T$, namely,

$$
P=\rho T /\left(\Gamma \mathrm{Ma}^{2}\right) .
$$

We arrange a semipermeable membrane panel with unbounded width in the $x_{2}$ and $x_{3}$ directions in the streamwise range of $0 \leqslant x_{1} / L \leqslant 0.05$, which is equivalent to introducing a friction term $-\alpha \rho U_{i}$ on the right-hand side of Eq. (3). In this paper, we choose

$$
\alpha= \begin{cases}\frac{25}{2}\left\{\left[1-\cos \left(2 \pi x_{2} / L\right)\right]\left[1-\cos \left(2 \pi x_{3} / L\right)\right]\right. & \text { if } 0 \leqslant x_{1} / L \leqslant 0.05 \\ \left.\quad+\left[1-\cos \left(4 \pi x_{2} / L\right)\right]\left[1-\cos \left(4 \pi x_{3} / L\right)\right]\right\}, & \text { otherwise }\end{cases}
$$

In this model, the friction coefficient varies in the $\left(x_{2}, x_{3}\right)$ plane with two spatial scales, $L$ and $L / 2$. This implies that the wake immediately behind the membrane will include only two dominant Fourier modes, which enables the analytical approximation in the following subsections.

At the inlet of the computational domain, the infinitesimal noises are chosen to be Gaussian, with a standard variation $0.001 U_{\infty}$ in the $x_{1}$ direction. A far-field boundary condition [18] is applied at the outlet of the domain, $x_{1}=16 \mathrm{~L}$. The Reynolds number is set as $\mathrm{Re}=10000$, while the Mach number is $\mathrm{Ma}=0.3$, indicating that the fluid can be approximately considered as incompressible (since the density change due to velocity is correspondingly about 5\%) [19]. This simulation is conducted by an in-house compressible finite difference method solver ASTR, which has been verified in various DNS cases [20,21]. A sixth-order compact central scheme [22] is used for the spatial discretization, and a third-order three-step total variation diminishing Runge-Kutta method [23] is used for time advance. The time step is set as 0.0006, determined by using the equation in Sec. 6.2.2 of Ref. [24] with CFL $=0.73$.

In order to quantitatively perform the following statistical analysis, we define \langle\rangle as the averaging over time $t$ and both spanwise directions $x_{2}$ and $x_{3}$. We note that the nature of this treatment, which involves spanwise average, is to consider the two large-scale structures as fluctuations rather than as mean shear. It is acceptable because (1) the present calculation is a simplification of grid-generated turbulence, which is always treated by spanwise averaging, and (2) as will be discussed later, according to the present definition, the skewness $S_{k}$ will include the information on the asymmetry of large-scale structures and allows the treatment of both laminar and turbulent flows in the same mathematical framework. Accordingly, the velocity fluctuation is defined as $u_{i}=U_{i}-\left\langle U_{i}\right\rangle$.

As shown in Fig. 2(a), we define three typical spanwise positions for the convenience of the following statistical analysis, i.e., $(L, L),(L / 2, L / 2)$, and $(L / 2, L)$ in the $\left(x_{2}, x_{3}\right)$ plane, respectively. They are denoted as positions 1,2 , and 3 , respectively. In each of these positions, we calculate the local dissipation scales $\eta$ at different $x_{1}$ locations, and compare them with the grid length $\Delta$ as shown in Fig. 2(b). It can be found that the maximum value of $\Delta / \eta$ is about 4.2 , which is consistent in magnitude with many existing DNS calculations [25-29] and supports the present mesh resolution. For example, according to the conclusion of Ref. [25], we would expect that the error of first- and second-order statistics is less than 5\%, and the error of third- and fourth-order statistics is less than $10 \%$ in the present calculation.

\section{B. DNS results}

The transition of the flow can be visually illustrated by using the instantaneous velocities. Figure 3 shows the snapshots of streamwise velocity $U_{1}$ at different $\left(x_{2}, x_{3}\right)$ planes; for completeness, the snapshot of $U_{1}$ at the $x_{2} / L=0.25$ plane [see the dashed line in Fig. 2(a) for a sketch] is 


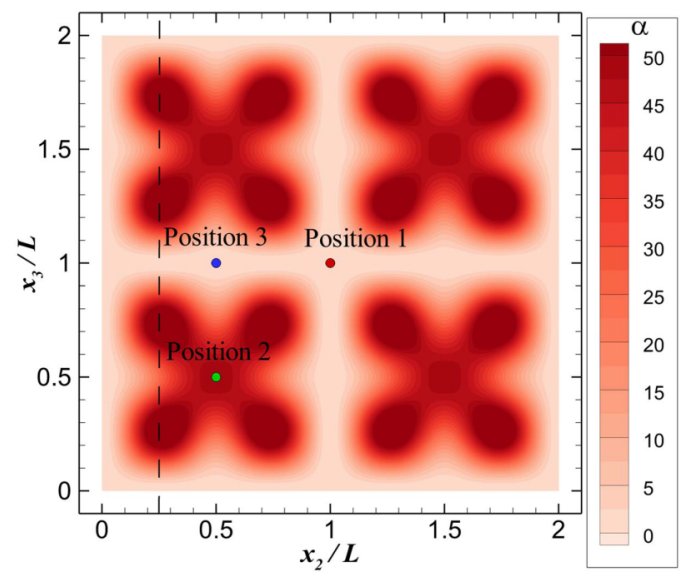

(a)

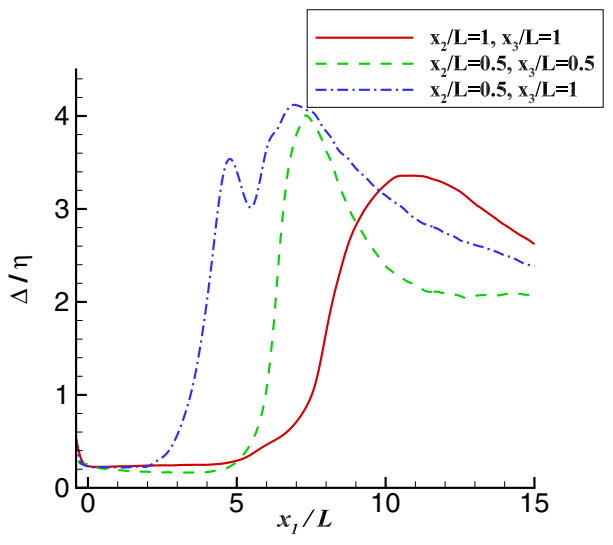

(b)

FIG. 2. Streamwise evolution of $\Delta / \eta$ at different positions in the two-scale wake, respectively. (a) The spanwise positions, denoted as 1,2 , and 3 , respectively. The dashed line indicates the location of $x_{2} / L=0.25$; (b) streamwise evolution of $\Delta / \eta$.

shown in Fig. 4. The $x_{1} / L=0.05$ plane [Fig. 3(a)] is the location just behind the membrane, while the streamwise velocity is redistributed due to the role of the friction term. The distribution of $U_{1}$ is quite similar to the friction coefficient $\alpha$, which is dominated by structures at two spatial scales $L$ and $L / 2$. As will be discussed in Sec. II C, this evolution of velocity redistribution also involves

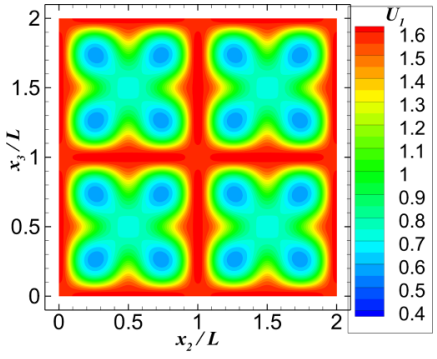

(a) $x_{1} / L=0.05$

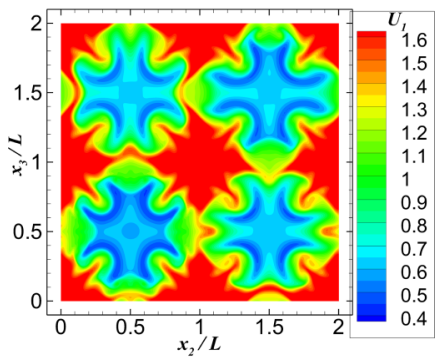

(d) $x_{1} / L=5.5$

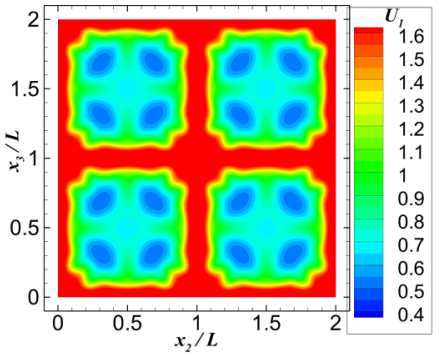

(b) $x_{1} / L=2$

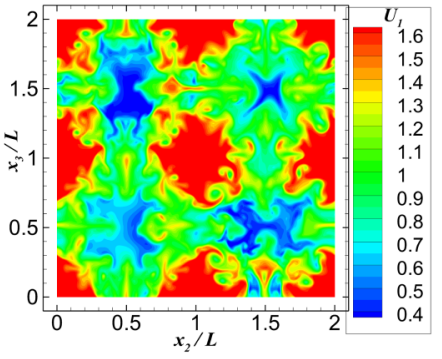

(e) $x_{1} / L=6.5$

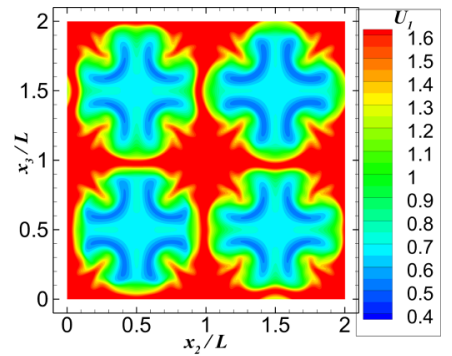

(c) $x_{1} / L=5$

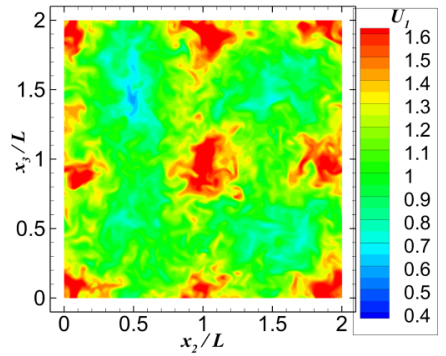

(f) $x_{1} / L=10.5$

FIG. 3. Snapshots of $U_{1}$ at different $\left(x_{2}, x_{3}\right)$ planes in the two-scale wake. 


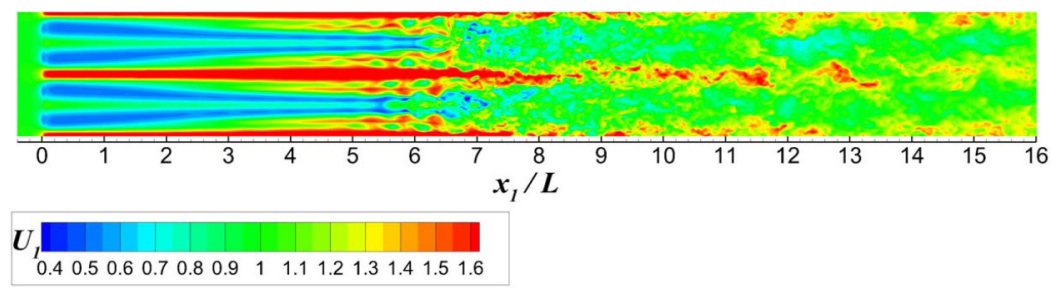

FIG. 4. A snapshot of $U_{1}$ at the $x_{2} / L=0.25$ plane in the two-scale wake.

spanwise velocities and generates streamwise vortices. Similar to the self-sustaining process in shear flows [30], the streamwise vortices feed the longitudinal streaks due to the lift-up mechanism. In Fig. 4 we can clearly observe these streaklike structures before $x_{1} / L=5$. The advection of these streaklike structures in spanwise directions involves more small-scale structures, as shown in Figs. 3(b)-(d). When $x_{1} / L=5$ [Fig. 3(c)], slight asymmetry can be observed, indicating that the small-scale perturbations are amplified because of the secondary instability, which is similar as the bypass transition in boundary-layer flows [31]. This instability finally yields turbulent flow structures, as shown in Figs. 3(e) and 3(f). The snapshot of $P$ at the $x_{2} / L=0.25$ plane is shown in Fig. 5, where it is clear that there is very weak adverse pressure gradient behind the membrane, while in the early transition region the pressure is quasiconstant.

We present the turbulence intensity $I$ and Taylor-scale Reynolds number $\operatorname{Re}_{\lambda}$ in Fig. 6. The rapidincreasing region of both them indicates the location of transition, which is about $4<x_{1} / L<7$, in agreement with the snapshots of Figs. 3 and 4.

As introduced in Sec. I, we focus on the skewness of streamwise velocity fluctuation gradient, defined as Eq. (1). As shown in Fig. 7, the value of $S_{k}$ is positive in the very early evolution before $x_{1} / L=3$, then it decreases rapidly to a negative number of about -1.7 and increases to an asymptotic value of about -0.5 . This asymptotic value -0.5 is in agreement with traditional turbulence theories [2,4], indicating that the flow can be considered to be fully developed in the region $x_{1} / L>7$. Before that, i.e., when $3<x_{1} / L<7$, the trend from decreasing to increasing is similar to Fig. 6 of Ref. [13], which may indicate that the flow in this region has some common properties as the nonequilibrium turbulence $[9,14,15]$. The most interesting phenomenon here would be the positive values of $S_{k}$ in the region $x_{1} / L<3$, i.e., the zone of early transition. We will introduce an analytical model to approximately explain this phenomenon in the next subsection.

\section{A simplified model for explaining the positive $S_{k}$}

In order to explain the phenomenon of positive $S_{k}$, we introduce a simplified model by using the following assumptions for the flow in the early transition stage:

(1) The flow is incompressible and inviscid. This was explained in the last subsection. Specifically, we remark that the inviscid assumption is appropriate since the flow in early transition is out

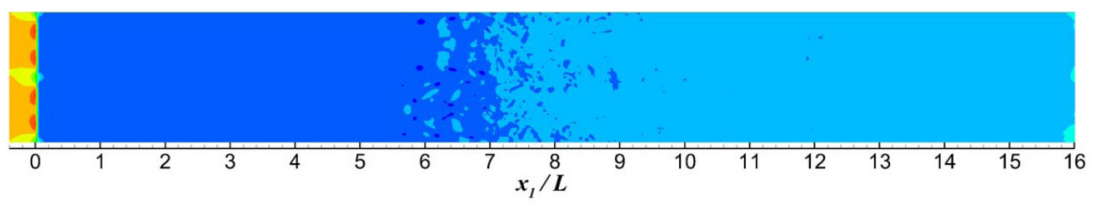

$\begin{array}{lllll}8 & 8.28 .48 .68 .8 \quad 9 & 9.29 .49 .69 .810\end{array}$

FIG. 5. A snapshot of $P$ at the $x_{2} / L=0.25$ plane in the two-scale wake. 


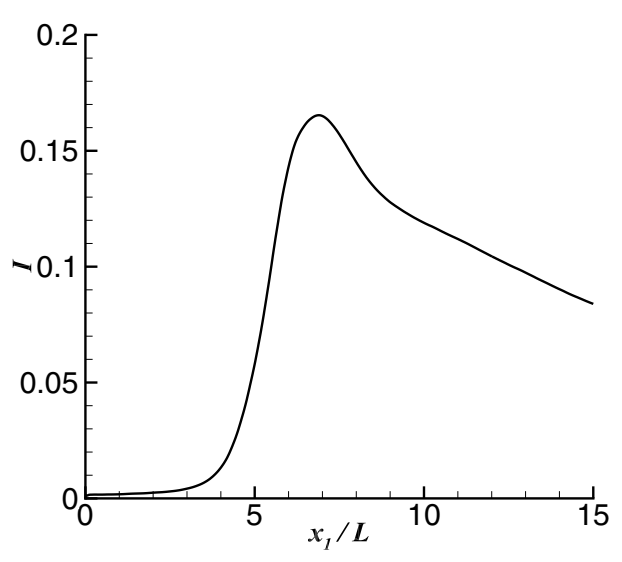

(a)

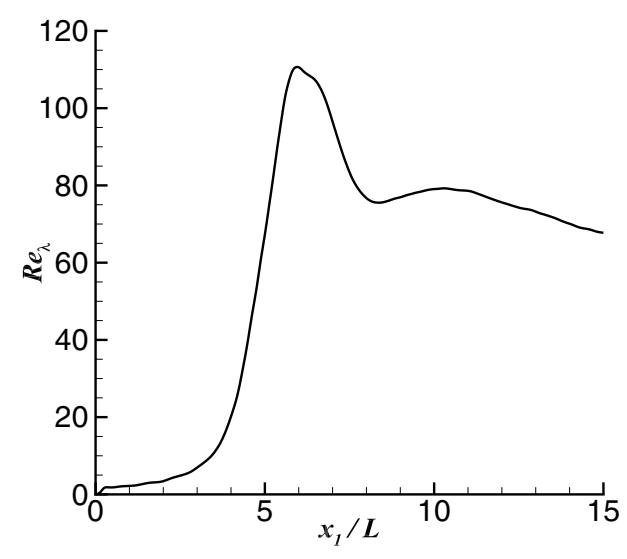

(b)

FIG. 6. Streamwise evolution of (a) turbulence intensity $I$ and (b) Taylor-scale Reynolds number $\operatorname{Re}_{\lambda}$ in the two-scale wake.

of equilibrium, while only advection is dominant. In fact, when the interscale energy transfer is well constructed, the viscosity effect should not be neglected [5,6,32,33].

(2) Symmetry between the $x_{2}$ and $x_{3}$ directions. This is reasonable since the distribution of $\alpha$ is symmetric.

(3) Similar to Ref. [30], we assume the decoupling between streamwise vortices and streamwise velocity. The underlying reason is that the high- and low-speed structures in early transition are analogous to the streaks in wall flows. This decoupling implies that the streamwise velocity is $U_{1}\left(x_{1}, x_{2}, x_{3}, t\right)$, but the spanwise velocities should be almost independent of $x_{1}$ and be written as $U_{2}\left(x_{2}, x_{3}, t\right)$ and $U_{3}\left(x_{2}, x_{3}, t\right)$, respectively. This assumption is verified in Fig. 8 by showing the spanwise velocities at different $x_{1}$ locations. It is observed that the main structures of streamwise

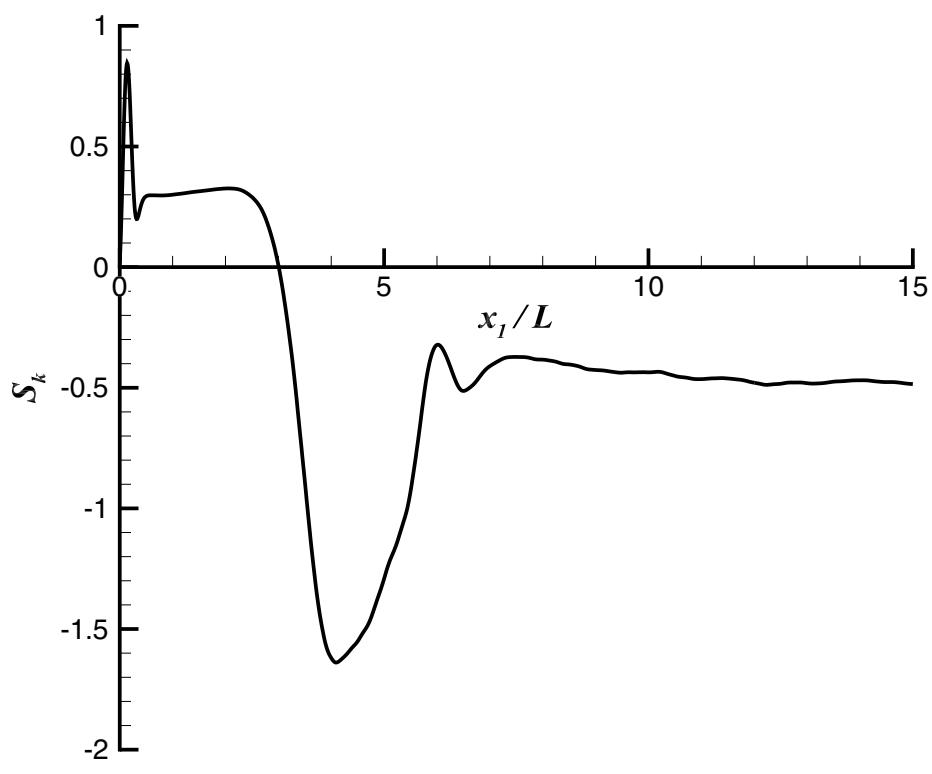

FIG. 7. Streamwise evolution of $S_{k}$ in the two-scale wake. 


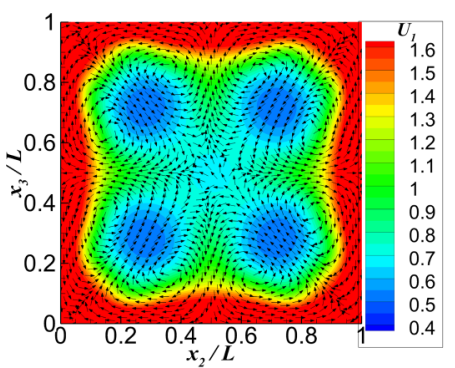

(a)

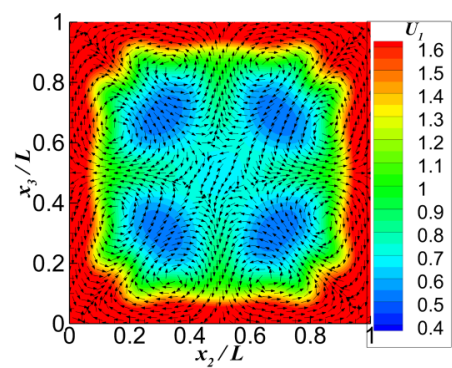

(b)

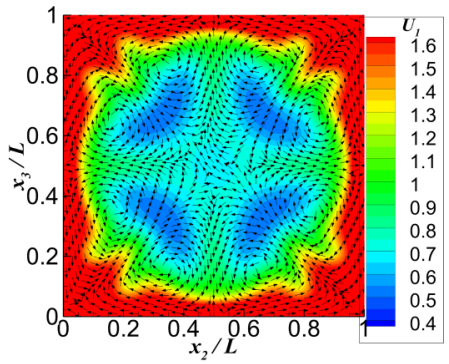

(c)

FIG. 8. Spanwise flow structures in the $L \times L$ cell at different $x_{1}$ locations in early transition of the twoscale wake, respectively. Arrows indicate the spanwise velocities, while contours are streamwise velocities. (a) $x_{1} / L=1$; (b) $x_{1} / L=2$; (c) $x_{1} / L=3$.

vortices at different $x_{1}$ locations are quite similar in Fig. 8, which can be sketched as Fig. 9. The full analytical expression will be discussed later.

(4) We assume that the streamwise velocity $U_{1}$ behind the membrane contains only the two largest scales, leading to the velocity profile

$$
\begin{aligned}
\left.U_{1}\right|_{x_{1} / L \geqslant 0.05}=a_{0}- & b\left[1-\cos \left(2 \pi x_{2} / L\right)\right]\left[1-\cos \left(2 \pi x_{3} / L\right)\right] \\
& \quad-c\left[1-\cos \left(4 \pi x_{2} / L\right)\right]\left[1-\cos \left(4 \pi x_{3} / L\right)\right] \\
= & a+b \cos \left(2 \pi x_{2} / L\right)+b \cos \left(2 \pi x_{3} / L\right)-b \cos \left(2 \pi x_{2} / L\right) \cos \left(2 \pi x_{3} / L\right) \\
& \quad+c \cos \left(4 \pi x_{2} / L\right)+c \cos \left(4 \pi x_{3} / L\right)-c \cos \left(4 \pi x_{2} / L\right) \cos \left(4 \pi x_{3} / L\right),
\end{aligned}
$$

where $a_{0}, a, b$, and $c$ are positive and evolute slowly along the streamwise location and $a=a_{0}-$ $b-c$, as shown in Fig. 10. The values of $b$ and $c$ correspond to the amplitude of the largest Fourier modes, respectively. To support this assumption, in Fig. 11 we show the streamwise evolution of perturbation energy and $\left(\partial u_{1} / \partial x_{1}\right)^{2}$ at different wave numbers, respectively. It is obvious that in the early transition region the first two wave numbers dominate, which correspond to the twoscale distribution of the friction coefficient. Higher wave numbers contain relatively less energy and dissipation, especially in the early transition range, indicating that they could be approximately neglected. According to Eq. (9) we have $b=c$ when $x_{1} / L=0.05$, which is verified in Fig. 10. In

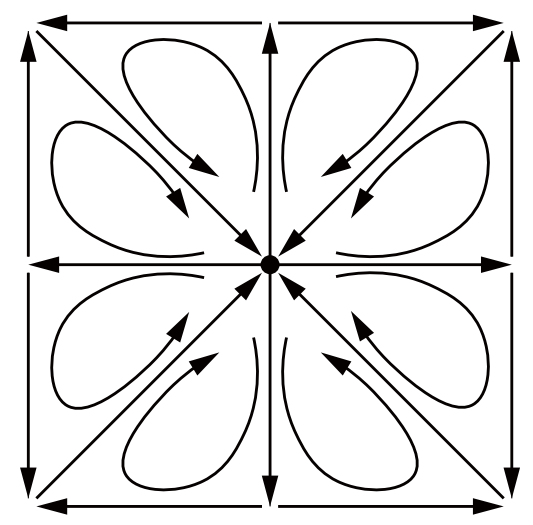

FIG. 9. Sketch of the main structures in the $L \times L$ cell of streamwise vortices in early transition of the two-scale wake. Arrows indicate the flow direction in a streamwise slice. 


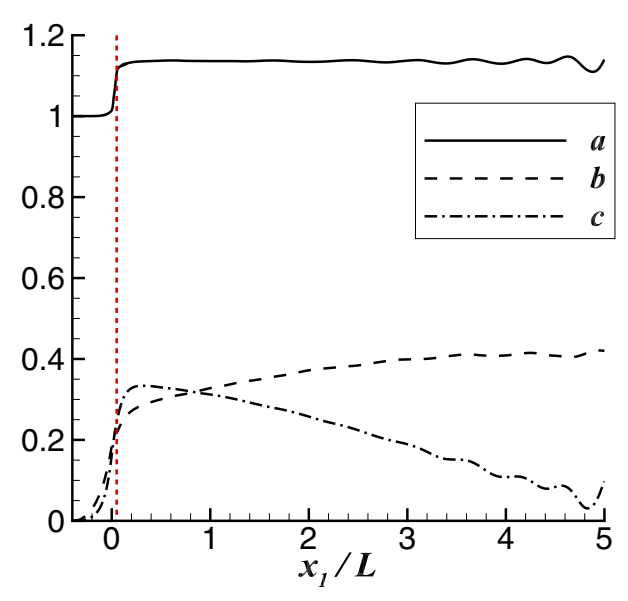

(a)

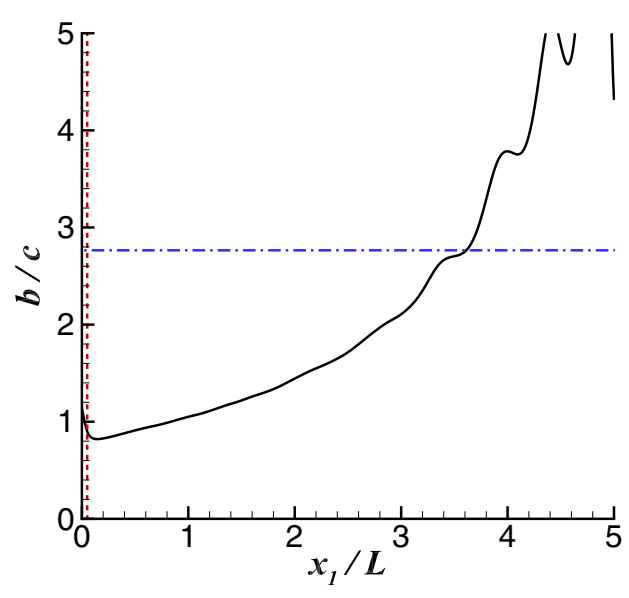

(b)

FIG. 10. The evolution of $a, b, c$, and $b / c$ along the streamwise direction in the two-scale wake. The red vertical line indicates the location behind the membrane $x_{1} / L=0.05$. The blue horizontal line corresponds to $b / c=2.765$, a critical value for the sign of $S_{k}$ according to our model.

the interval $0.05<x_{1} / L<5$, The evolution trends of $b$ and $c$ in Fig. 10 are similar to the curves $k=1$ and $k=2$ in Fig. 11(a), respectively, since they both describe the energy at large scales. The value of $a$ corresponds to the mean streamwise velocity, which increases from 1 to about 1.12 behind the membrane. This increase corresponds to the weak compressibility. In the early transition stage behind the membrane, it is shown in Fig. 10 that $a$ is quasiconstant due to the zero pressure gradient, indicating the assumption

$$
\frac{\partial\left\langle U_{1}\right\rangle}{\partial x_{1}}=0
$$

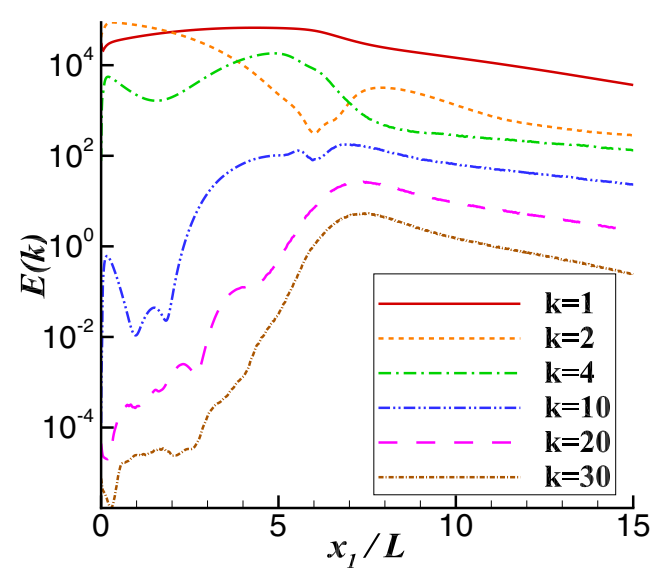

(a)

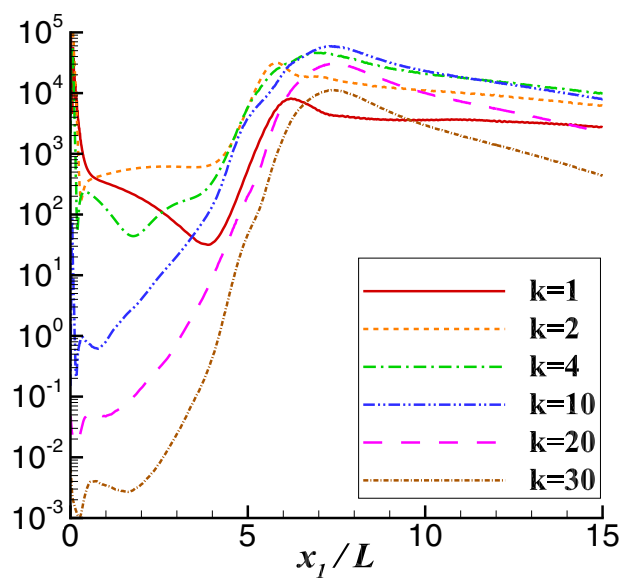

(b)

FIG. 11. Streamwise evolution of (a) kinetic energy and (b) $\left(\partial u_{1} / \partial x_{1}\right)^{2}$ at several wave numbers in the two-scale wake. The wave number in normalized by $2 \pi / L$. 
This assumption is a weak version of Taylor's frozen-flow hypothesis, which has been widely used in experimental studies of grid turbulence [34]. Note that we do not assume constant $b$ or $c$.

(5) Also, we assume that the velocity profiles of $U_{2}$ and $U_{3}$ contain only the two largest scales.

To introduce the model in the early transition region, we start from the advection equation

$$
U_{1} \frac{\partial U_{1}}{\partial x_{1}}+U_{2} \frac{\partial U_{1}}{\partial x_{2}}+U_{3} \frac{\partial U_{1}}{\partial x_{3}}=0
$$

Comparing to the advection-diffusion equation (1) of Ref. [30], here we neglect the diffusion because of assumption (1). There is no pressure term in Eq. (12) because of the following two facts: (1) As shown in Fig. 5, in the early transition region the pressure is quasiconstant. (2) As discussed in Ref. [30], the pressure effect is not important for the generation of streaks by streamwise vortices (this can be explained by the lift-up mechanism without the attendance of pressure gradient), although it plays a role in the formation of small-scale waves due to instability. Because in the early transition region the instability waves have not been accumulated to finite level, the pressure effect is neglected. From assumption (4), we have locally $\frac{\partial\left\langle U_{1}\right\rangle}{\partial x_{1}}=0$, hence $\frac{\partial U_{1}}{\partial x_{1}}=\frac{\partial u_{1}}{\partial x_{1}}$. In addition, in the present case $U_{1}$ is always positive at any position in the calculation domain, leading to the relation

$$
\operatorname{sgn}\left(S_{k}\right)=\operatorname{sgn}\left\langle\left(U_{1} \frac{\partial U_{1}}{\partial x_{1}}\right)^{3}\right\rangle=\operatorname{sgn}\left\langle\left(-U_{2} \frac{\partial U_{1}}{\partial x_{2}}-U_{3} \frac{\partial U_{1}}{\partial x_{3}}\right)^{3}\right\rangle .
$$

Here $U_{1}$ is assumed to satisfy the expression (10). Therefore, in order to explain the phenomenon of positive $S_{k}$, a key question is to write the expressions of spanwise velocities $U_{2}$ and $U_{3}$.

Following Waleffe [30], the distribution of spanwise velocities should be a solution of the lefthand side of Eq. (6) in Ref. [30]. As a consequence, for $U_{2}\left(x_{2}, x_{3}, t\right)$ we write

$$
\left(\frac{\partial}{\partial t}-\frac{1}{\operatorname{Re}} \nabla^{2}\right) \nabla^{2} U_{2}\left(x_{2}, x_{3}, t\right)=0
$$

For the convenience of writing the solutions, we define a coordinate transformation $\xi_{2}=x_{2}-$ $L / 2, \xi_{3}=x_{3}-L / 2$, and rewrite Eq. (14) as

$$
\left(\frac{\partial}{\partial t}-\frac{1}{\operatorname{Re}} \nabla^{2}\right) \nabla^{2} U_{2}\left(\xi_{2}, \xi_{3}, t\right)=0
$$

Due to periodicity and symmetry (see Fig. 9 for the sketch), $U_{2}\left(\xi_{2}, \xi_{3}, t\right)$ should be symmetric with respect to the $\xi_{3}$ axis and antisymmetric with respect to the $\xi_{2}$ axis. The boundary conditions are

$$
U_{2}\left( \pm L / 2, \xi_{3}, t\right)=0 .
$$

For $U_{3}\left(\xi_{2}, \xi_{3}, t\right)$ the description is similar.

On another hand, due to the decoupling with streamwise velocity [assumption (3)], the continuity equation writes

$$
\frac{\partial U_{2}\left(\xi_{2}, \xi_{3}, t\right)}{\partial \xi_{2}}+\frac{\partial U_{3}\left(\xi_{2}, \xi_{3}, t\right)}{\partial \xi_{3}}=0
$$

As shown in the Appendix for the derivation details, for all two-scale solutions [assumption (5)] of Eqs. (15) and (17), the only symmetric solution [assumption (2)] should be written as

$$
\left\{\begin{array}{l}
U_{2}\left(\xi_{2}, \xi_{3}\right)=-d \sin \left(2 \pi \xi_{2} / L\right) \cos \left(4 \pi \xi_{3} / L\right)+\frac{d}{2} \sin \left(4 \pi \xi_{2} / L\right) \cos \left(2 \pi \xi_{3} / L\right), \\
U_{3}\left(\xi_{2}, \xi_{3}\right)=-d \sin \left(2 \pi \xi_{3} / L\right) \cos \left(4 \pi \xi_{2} / L\right)+\frac{d}{2} \sin \left(4 \pi \xi_{3} / L\right) \cos \left(2 \pi \xi_{2} / L\right),
\end{array}\right.
$$

with $d$ positive constant. The velocity field is accordingly shown in Fig. 9. 
The sign of $S_{k}$ can be evaluated by substituting Eqs. (10) and (18) into Eq. (13). We obtain

$$
\operatorname{sgn}\left(S_{k}\right)=\operatorname{sgn}\left(-b c^{2} d^{3}\left[20\left(\frac{b}{c}\right)^{2}+289 \frac{b}{c}-952\right]\right) .
$$

It is then easy to obtain $S_{k}>0$ when $b / c<2.765$, while $S_{k}<0$ when $b / c>2.765$. In the present DNS case, as shown in Fig. 10, we have $b / c=1$ just behind the membrane; therefore $S_{k}$ is positive, corresponding to the phenomenon in DNS.

This result can be further used to predict the streamwise interval for positive $S_{k}$. According to Fig. 10(b), the value of $b / c$ increases from 1 to 2.765 in the streamwise interval $x_{1} / L<3.8$, which corresponds to positive $S_{k}$ from the above model. This interval is close to the DNS evidence in Fig. 7, where positive $S_{k}$ corresponds to about $x_{1} / L<3$.

We can remark on the present model from the viewpoint of energy transfer. If the flow is dominated by only two largest scales, the sign of $S_{k}$ is determined by the ratio of the energies at the two scales, i.e., $b / c$. If initially the smaller scale contains enough energy, i.e., $c$ is not too small, then $b / c$ can be less than 2.765 and the value of $S_{k}$ is positive. On the other hand, we know that positive $S_{k}$ usually indicates the backward energy transfer in turbulence [2,14], thus analogically it is reasonable in the present case that the smaller scale transfers energy towards the larger scale, i.e., $c$ decreases, $b$ increases, and $b / c$ increases. Finally $b / c$ increases to greater than the critical value 2.765 , yielding negative $S_{k}$, which corresponds to forward energy transfer.

The present model illustrates that the energies, or, e.g., amplitudes, at the two largest scales can affect the sign of $S_{k}$. This mechanism will be supported by another different flow example in the next section.

\section{TRANSITION IN A TWO-DIMENSIONAL RAYLEIGH-TAYLOR FLOW WITH A DIFFUSE INTERFACE}

\section{A. Physical model}

In this section, we will show another physical problem to illustrate the phenomenon of positive $S_{k}$. The physical model to be studied is a two-dimensional inviscid Rayleigh-Taylor (RT) problem occurring at a diffuse interface of two miscible fluids with different densities (see Ref. [35] for details). The two-dimensional Cartesian coordinate system $(x, y)$ is employed, with the origin $o$ at the interface and $x$ and $y$ perpendicular and parallel to the interface. The positive $x$ is towards the light fluid, so is the acceleration $g$. The density disparity is characterized by a dimensionless parameter, the Atwood number, which is defined as $A=\left(\rho_{h}-\rho_{l}\right) /\left(\rho_{h}+\rho_{l}\right)$, where $\rho_{h}$ and $\rho_{l}$ are the densities of the heavy and light fluids, respectively. The length scales $(x, y)$, time $t$, velocity field $\mathbf{U}=(u, v)$, density $\rho$, and pressure $p$ are normalized by $L_{m}, \sqrt{L_{m} / g}, \sqrt{g L_{m}}, \rho_{h}$, and $\rho_{h} g L_{m}$, respectively, where $L_{m}=\min \left[\rho_{0}^{*} /\left(d \rho_{0}^{*} / d x^{*}\right)\right]$ is the minimum density gradient scale length, and the asterisk represents the dimensional quantities. The flow is governed by dimensionless Eulerian equations, which are the same as (2) with the body force and viscous terms neglected. Note that the present model is based on the inertial confinement fusion engineering, for which the temperature is extremely high such that all the gas molecules are dissociated. Thus, the perfect-gas model is also employed, but in the equation of the state (8), the ratio of the specific heat $\Gamma$ is referred to as the adiabatic exponent, with its value being changed to $5 / 3$.

At the beginning, the flow field is a sum of a base flow which satisfies the steady Eulerian equation and an infinitesimal perturbation,

$$
(\rho, u, v, p)=\left(\rho_{0}, 0,0, p_{0}\right)(x)+\epsilon_{0}\left(\exp \left(-x^{2}\right), 0,0,0\right) \cos (k y),
$$

where $\epsilon_{0} \ll 1$ denotes the initial amplitude of the perturbation, and $k$ the wave number in the direction tangential to the interface. The density and pressure of the base flow are selected as

$$
\rho_{0}(x)=[1-A \tanh (\delta x)] /(1+A),
$$




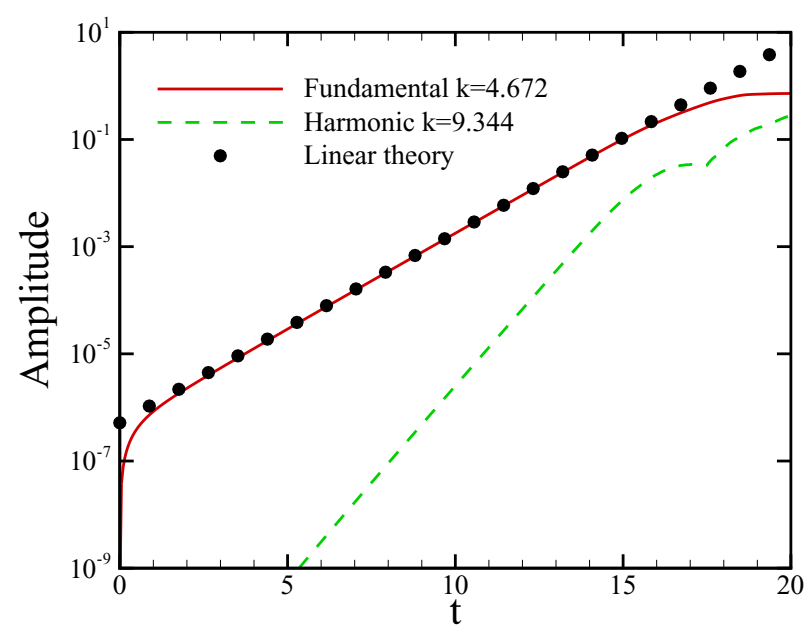

FIG. 12. Evolution of the amplitude of the fundamental $(k=4.672)$ and harmonic $(k=9.344)$ perturbations in the two-dimensional RT flow.

$$
p_{0}(x)=p_{c}+\int_{x_{c}}^{x} \rho_{0}(x) d x
$$

where $\delta=A /\left[2\left(1-\sqrt{1-A^{2}}\right)\right]$ is a dimensionless coefficient such that $\min \left[\rho_{0} /\left(d \rho_{0} / d x\right)\right]=1$ and $p_{c}$ is a reference pressure at a reference position $x_{c}$. For the diffuse interface, $\delta^{-1}$ characterizes the thickness of the density-adjustment layer. In this paper, we choose $\left(p_{c}, x_{c}\right)=(45,-15), A=0.4$, $\epsilon_{0}=10^{-6}$, and $k=4.672$, the same as the parameters of case 1 in Ref. [35]. In the simulation, the computational domain is selected as $[-10,10] \times\left[0, y_{l}\right]$, with $2001 \times 121$ grid points employed, where $y_{l}=2 \pi / k \approx 1.34$.

\section{B. Numerical results}

The temporal evolution of the perturbation starting from (20) is obtained by DNS. The amplitude of the fundamental perturbation is shown by the red solid line in Fig. 12. As revealed by Refs. [36,37], the RT instability for a diffuse interface admits an infinite number of discrete eigenmodes with different growth rates. An arbitrarily introduced initial perturbation [the second term on the right-hand side of (20)] is considered as a superposition of all the eigenmodes, whose amplitudes can be obtained by the multimode decomposition as in [35]. At early time instants, $t<2$, all of these eigenmodes are competing, rendering an algebraic growth of the total amplitude, which was referred to as a prelinear phase. Then the dominant eigenmode (with the greatest growth rate) amplifies exponentially, with its growth rate obtained by the slope of the amplitude curve, 0.817 , which agrees with the prediction of the linear stability theory (the black circles). After $t \approx 15$, the amplitude tends to be saturated due to nonlinearity, which is referred to as the nonlinear saturation phase. In this phase, the bubble-spike structure, as shown in Fig. 13, appears and grows. At ever later time instants, the small-scale structures appear due to the Kelvin-Helmholtz instability regime, which eventually leads to transition to turbulent mixing.

The dashed line in Fig. 12 denotes the amplitude of the second-order harmonic perturbation, whose wave number is twice of the fundamental wave number. Before $t=15$, the harmonic perturbation amplifies exponentially, with the growth rate being about twice of that of the fundamental one. 


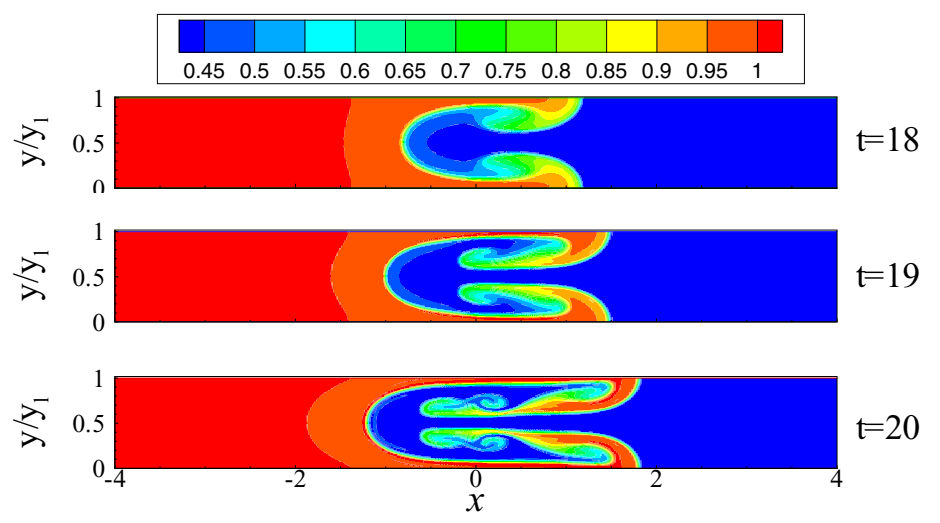

FIG. 13. Contours of the density at $t=18,19$ and 20 for the DNS result of the two-dimensional RT flow, respectively.

Since the base flow is stationary, the instantaneous velocity $u$ is also the perturbation to the base flow. We define the ensemble \langle\rangle as the spatial averaging over the $y$ direction. According to Eq. (1), it is easy to see that in the linear phase, for which the perturbation is sinusoidal with a single wave number in $y$, the skewness is exactly zero. However, if the flow field includes more length scales with moderate amplitudes in the $y$ direction, $S_{k}$ may be nonzero. Such a situation can occur when a single-mode RT instability evolves to the weakly nonlinear phase, which is almost the most simple setup of the RT instability simulations.

Figure 14 shows the values of $S_{k}$ in the $x$ - $t$ plane. $S_{k}$ is exactly zero in the early time, but from $t \approx 11$ to 15 , positive skewness can be observed around the interface $x \approx 0$. Intuitively, this should be due to the weakly nonlinear growth of the harmonic mode. As time advances, higher-order harmonics are excited and reach finite amplitude, rendering a more complicated distribution of $S_{k}$. Additionally, two strips with negative $S_{k}$ are located at the edges of the spike and bubble, which expand as time advances.

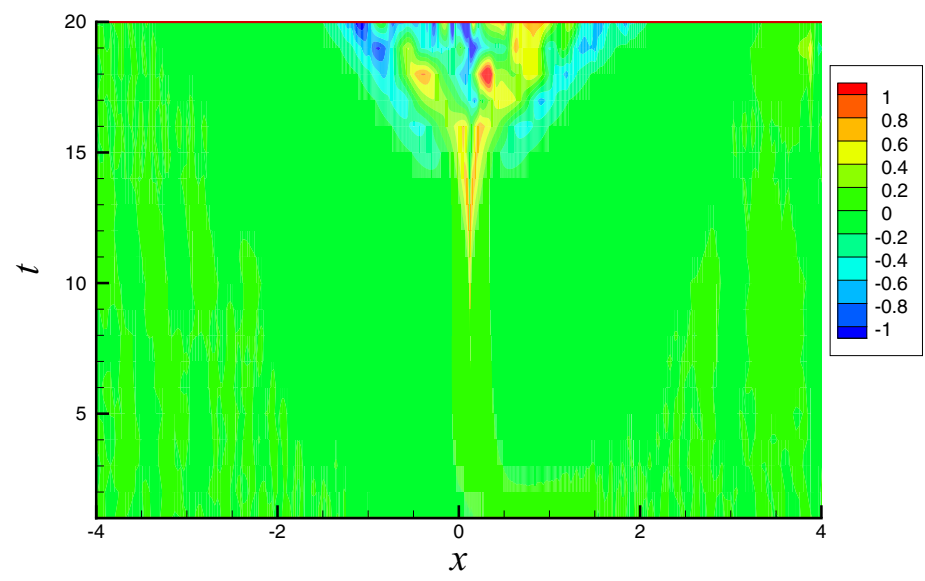

FIG. 14. Contours of the skewness $S_{k}(x, t)$ in the two-dimensional RT flow. 


\section{Prediction of the skewness using the weakly nonlinear theory}

In the weakly nonlinear phase, when the fundamental mode reaches a finite amplitude, the highorder harmonics are exited in sequence. Now our attention is restricted in the early nonlinear stage, in which only the second-order harmonic mode comes into play, and the feedback from the growth of the harmonic mode to the fundamental one, as well as to the base flow, is negligible. The perturbation field is therefore expressed as

$$
\varphi=\varphi_{0}(x)+\epsilon \hat{\varphi}_{1}(x) e^{\gamma t+i k y}+\epsilon^{2} \hat{\varphi}_{2}(x, t) e^{2 i k y}+\cdots+\text { c.c. },
$$

where $\varphi_{0}=\left(\rho_{0}, 0,0, p_{0}\right)^{T}, \hat{\varphi}=(\hat{\rho}, \hat{u}, \hat{v}, \hat{p})^{T}, i=\sqrt{-1}, \epsilon$ is the amplitude of the excited RT instability mode, and c.c. represents the complex conjugate. $\epsilon$ can be obtained by the multimode decomposition as in Ref. [35], and for the present configuration, $\epsilon=1.02 \epsilon_{0}$.

Following Ref. [37], the eigenfunction of the fundamental mode for a high wave number $k \gg 1$ (in the present setup, $k=4.672$ is already sufficient to be approximated as $k \gg 1$, as proven by Ref. [37]) can be described by the Wentzel-Kramers-Brillouin approximation, and the perturbation velocity $\hat{u}_{1}$ reads

$$
\hat{u}_{1} \sim \sin \left(k \int_{x_{B}}^{x} \sqrt{G_{0}} d x+\frac{\pi}{4}\right), \quad x \in\left(x_{B}, x_{A}\right)
$$

where

$$
G_{0}=\left(\frac{\rho_{0}}{p_{0} \Gamma}-\frac{\rho_{0}^{\prime}}{\rho_{0}}\right) / \gamma^{2}-1,
$$

and $x_{B}$ and $x_{A}$ are the two zero (turning) points of $G_{0}$. For mode- 1 perturbation, the most unstable mode,

$$
k \int_{x_{B}}^{x_{A}} \sqrt{G_{0}} d x=\frac{\pi}{2} .
$$

Note that in the regions $x<x_{B}$ and $x>x_{A}$, the eigenfunction dampens to zero exponentially. The variations of the base flow $\rho_{0}$ and $p_{0}$ are much slower than the exponential (sin) behavior of the perturbation. In the following, the analysis will be performed at different local positions with a rather short length scale $x$, such that the variations of the base flow are negligible.

From the linearized Eulerian equation, we obtain the perturbation pressure, density, and tangential velocity in terms of asymptotic series,

$$
\begin{aligned}
& \hat{p}_{1}=\frac{\hat{p}_{10}}{k}+\frac{\hat{p}_{11}}{k^{2}}+O\left(k^{-3}\right), \\
& \hat{\rho}_{1}=\hat{\rho}_{10}+\frac{\hat{\rho}_{11}}{k}+O\left(k^{-2}\right), \\
& \hat{v}_{1}=\hat{v}_{10}+\frac{\hat{v}_{11}}{k}+O\left(k^{-2}\right),
\end{aligned}
$$

where

$$
\begin{gathered}
\hat{p}_{10}=-\gamma \rho_{0} \sqrt{G_{0}} \cos \Theta, \quad \hat{p}_{11}=-\frac{\gamma \rho_{0}^{2}}{\Gamma p_{0}} \sin \Theta \\
\hat{\rho}_{10}=\frac{1}{\gamma}\left(\frac{\rho_{0}^{2}}{\Gamma p_{0}}-\rho_{0 x}\right) \sin \Theta, \quad \hat{\rho}_{11}=\frac{-\gamma \rho_{0}^{2} \sqrt{G_{0}}}{\Gamma p_{0}} \cos \Theta, \\
\hat{v}_{10}=i \sqrt{G_{0}} \cos \Theta, \quad \hat{v}_{11}=\frac{i \rho_{0}}{\Gamma p_{0}} \sin \Theta
\end{gathered}
$$


with $\Theta=k \int_{x_{B}}^{x} \sqrt{G_{0}} d x+\frac{\pi}{4}$.

The second-order harmonic behaves like

$$
\hat{\varphi}(x, t)=\Phi(t) \exp \left(2 k \int_{x_{B}}^{x} \sqrt{G_{0}} d x+\frac{\pi}{2}\right)+\text { c.c. }
$$

where $\Phi=\left(\Phi_{\rho}, \Phi_{u}, \Phi_{v}, \Phi_{p}\right)^{T}$. Substituting into the Eulerian equations, and collecting the leadingorder terms, we obtain

$$
\begin{array}{r}
\Phi_{\rho}^{\prime}+2 i k \rho_{0}\left(\sqrt{G_{0}} \Phi_{u}+\Phi_{v}\right)+\rho_{0, x} \Phi_{u}=-A_{1} e^{2 \gamma t}, \\
\rho_{0} \Phi_{u}^{\prime}+2 i k \sqrt{G_{0}} \Phi_{p}-\Phi_{\rho}=-A_{2} e^{2 \gamma t}, \\
\rho_{0} \Phi_{v}^{\prime}+2 i k \Phi_{p}=-A_{3} e^{2 \gamma t}, \\
\Phi_{p}^{\prime}+\rho_{0} \Phi_{u}+2 i k \Gamma p_{0}\left(\sqrt{G_{0}} \Phi_{u}+\Phi_{v}\right)=-\frac{A_{4}}{k} e^{2 \gamma t},
\end{array}
$$

where

$$
A_{1}=\frac{\rho_{0}}{2 \gamma \Gamma p_{0}}\left(\frac{\rho_{0}^{2}}{\Gamma p_{0}}-\rho_{0, x}\right), \quad A_{2}=\frac{\rho_{0, x}}{4}, \quad A_{3}=-\frac{\sqrt{G_{0}} \rho_{0, x}}{4}, \quad A_{4}=-\frac{i \gamma \sqrt{G_{0}}(1+\Gamma) \rho_{0}^{2}}{4 \Gamma p_{0}} .
$$

Eliminating $\Phi_{\rho, v, p}$, and noting that $k \gg 1$, we arrive at

$$
\Phi_{u}^{\prime \prime}-F_{1} \Phi_{u}=F_{0} e^{2 \gamma t}
$$

where

$$
F_{1}=\frac{1}{G_{0}+1}\left(\frac{\rho_{0}}{p_{0} \Gamma}-\frac{\rho_{0}^{\prime}}{\rho_{0}}\right), \quad F_{0}=-\frac{A_{1}+2 \gamma A_{2}-2 A_{3} \gamma \sqrt{G_{0}}}{\left(G_{0}+1\right) \rho_{0}} .
$$

Taking into account (25), we obtain

$$
F_{1}=\gamma^{2}, \quad F_{0}=-\frac{1}{2 \gamma \rho_{0}}\left(\frac{\rho_{0}}{p_{0} \Gamma}-\frac{\rho_{0}^{\prime}}{\rho_{0}}\right) .
$$

Note that $F_{1}$ is positive, and $F_{0}$ is negative at all positions of our interest. The solution for $\Phi_{u}$ is

$$
\Phi_{u}=\frac{F_{0}}{3 \gamma^{2}} e^{2 \gamma t}+C_{1} e^{\gamma t}+C_{2} e^{-\gamma t}
$$

where $C_{1}$ and $C_{2}$ are constants. We will focus on the particular part with a growth rate of $2 \gamma$, as confirmed by the dashed line in Fig. 12.

Now we can express the instantaneous velocity as

$$
u=\epsilon \bar{A} \cos (k y)+\epsilon^{2} \bar{B} \cos (2 k y),
$$

where $\bar{A}=\sin \Theta e^{\gamma t}$ and

$$
\bar{B}=\left(F_{0} / 3 \gamma^{2}\right) \sin (2 \Theta) e^{2 \gamma t}
$$

Figure 15 plots the eigenprofiles of the fundamental and second-order harmonic perturbations at representative time instants in the weakly nonlinear phase, the simplified profiles (33) are overall 


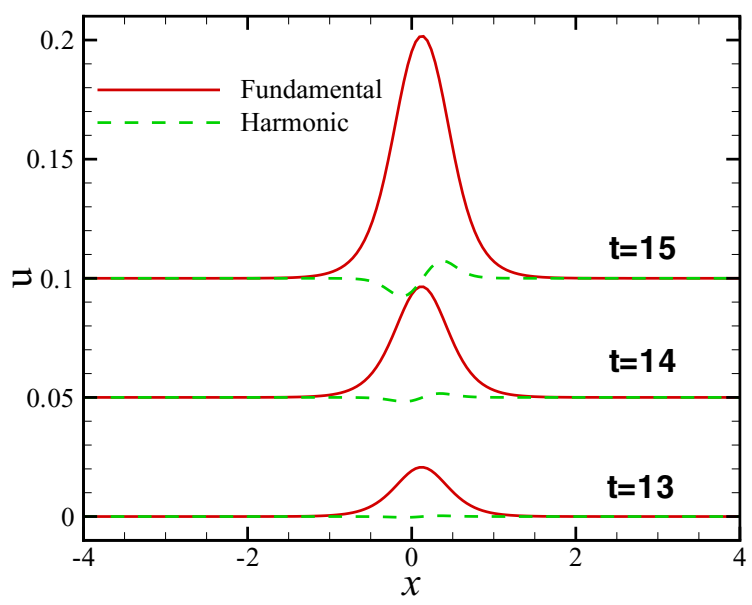

FIG. 15. Eigenprofiles of the fundamental and second-order harmonic perturbations for different $t$ in the two-dimensional RT flow. The curves for $t=14$ and 15 are shifted for plotting convenience.

confirmed. Then the skewness at a time instant $t_{0}$ is expressed as

$$
\begin{aligned}
S_{k}\left(x, t_{0}\right) & =\frac{1}{y_{l} \bar{C}} \int_{0}^{y_{l}}\left(\frac{\partial u}{\partial x}\right)^{3} d y \\
& =\frac{1}{y_{l} \bar{C}} \int_{0}^{y_{l}}\left[\epsilon \bar{A}_{x} \cos (k y)+\epsilon^{2} \bar{B}_{x} \cos (2 k y)\right]^{3} d y \\
& =\frac{1}{y_{l} \bar{C}} \epsilon^{3} \bar{A}_{x}^{2} \bar{B}_{x} \int_{0}^{y_{l}} \cos ^{2}(k y) \cos (2 k y) d y \\
& =\frac{1}{y_{l} \bar{C}} \epsilon^{3} \bar{A}_{x}^{2} \bar{B}_{x} \int_{0}^{y_{l}} \frac{1}{2} \cos ^{2}(2 k y) d y=\frac{\epsilon^{3} \bar{A}_{x}^{2} \bar{B}_{x}}{4 \bar{C}},
\end{aligned}
$$

where the subscript $x$ denotes partial derivative in the $x$ direction, and $\bar{C}>0$ represents the denominator of $S_{k}$. Because $\bar{A}_{x}^{2}$ and $\bar{C}$ are non-negative, the sign of $S_{k}$ depends only on the behavior of $\bar{B}_{x}$. It is obtained from (34) that

$$
\operatorname{sgn}\left[\bar{B}_{x}\right]=\operatorname{sgn}\left[\frac{2 F_{0} \sqrt{G_{0}} k}{3 \gamma^{2}} \cos (2 \Theta)\right]=\operatorname{sgn}[-\cos (2 \Theta)] .
$$

In the interval of our interest, $x \in\left(x_{B}, x_{A}\right)$, because the cos function changes its phase from $\pi / 2$ to $3 \pi / 2$, which is always negative, $\bar{B}_{x}$ is positive, so is the skewness $S_{k}$. However, in the regions $x<x_{B}$ and $x>x_{A}, S_{k}$ could become negative. As shown in Fig. 14, in the weakly nonlinear phase, $10<t<15, S_{k}$ is positive around the interface $x=0$, but negative in the outer regions, $x<x_{B}$ and $x>x_{A}$. This is exactly in agreement with the above theoretical predictions.

Figure 16 further compares the theoretical prediction of the skewness with the numerical results for different time instants, $t=11,13$, and 14, where the skewness is normalized by its maximum value $S_{k, \max }=\max _{x}\left(S_{k}\right)$. The distributions of different curves around the interface $x=0$ agree well, confirming our weakly nonlinear analysis. Note that the distributions of $\bar{A}_{x}^{2}$ and $\bar{C}$ are quite similar, the normalized skewness can be approximated by $S_{k} / S_{k, \max } \approx \bar{B}_{x} / \max _{x}\left(\bar{B}_{x}\right)$, confirming the significance of $\bar{B}_{x}$ on predicting the sign of $S_{k}$. In later times, the single-peak distribution of $S_{k}$ is replaced by a multipeak distribution, due to the excitation of higher-order harmonics, which is not shown, however, because it is unrelated to our topic.

We remark that the present model shows the similar mechanism for the phenomenon of positive $S_{k}$ as the two-scale wake case. Here the relation of the amplitudes of harmonic mode is much smaller 


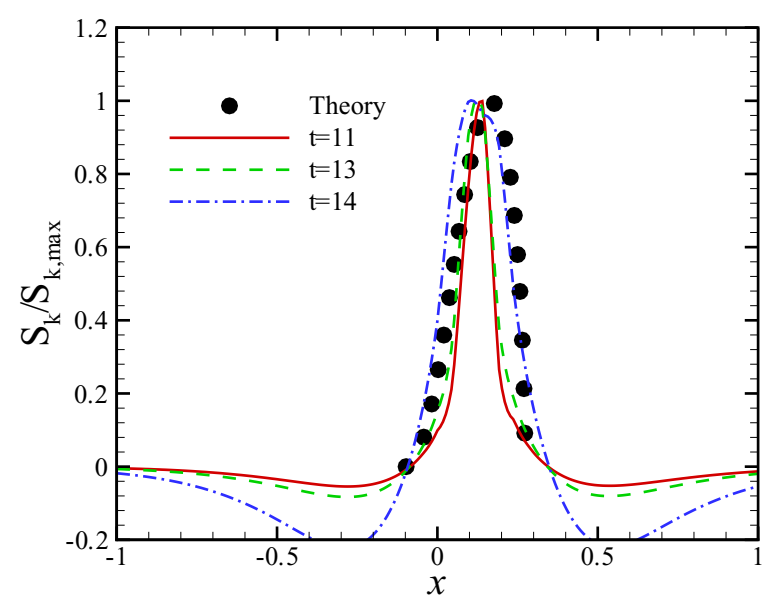

FIG. 16. Comparison of normalized skewness by its maximum value, $S_{k} / S_{k, \max }$, between the theoretical prediction and the simulation results.

than the fundamental mode, characterized by $\bar{B}_{x}$, is found to be the main origin of the positive $S_{k}$ phenomenon in Eq. (36). In the next section we will give more explanations and perspectives.

\section{CONCLUDING REMARKS}

In the statistical point of view, the skewness of the longitudinal velocity gradient $S_{k}$ is usually related to energy transfer in turbulence; for example, the Kolmogorov forward energy cascade in fully developed turbulence implies negative $S_{k}$, while Gaussian random noise and infinitesimal perturbations lead to zero $S_{k}$. In this sense, revealing more about the evolution of $S_{k}$ will be helpful for understanding the formation of the equilibrium turbulence, which is undoubtedly a key unclear question till now. In the present contribution, we have shown by two DNS cases that, in the early transition stage, $S_{k}$ can be positive, in contrast to the energy cascade in equilibrium turbulence.

Two analytical models, introduced in Secs. II C and III C, can predict the phenomenon of positive $S_{k}$, which is in good agreement with the DNS results. Both models assume that the flow is constituted by only two dominant Fourier modes with harmonic relations. Although this is a very simple simplification, the phenomenon of positive $S_{k}$ is well captured. Both analytical models show that $S_{k}$ can be positive or negative, according to the different interactions between these two Fourier modes. In both flows, the sign of $S_{k}$ is attributed to the amplitudes of the largest modes. We remark that this does not mean that the relation of amplitudes is the only mechanism. In fact, the effect of amplitudes was obtained by fixing the relation of velocity phases in the present paper. For example, the distribution function (9) of $\alpha$ implies that the velocity phases at the two largest scales are aligned. We will show that the phase relation can also affect the sign of $S_{k}$. Taking a two-dimensional flow as an example, if the longitudinal velocity component $u_{1}$ can be expressed in terms of Fourier series, i.e., $u_{1}(\vec{x})=\sum_{\vec{k}} e^{i \vec{k} \cdot \vec{x}} a_{1}(\vec{k}) e^{i \phi_{1}(\vec{k})}+$ c.c., simple derivations will lead to the expression

$$
\left\langle\left(\frac{\partial u_{1}}{\partial x_{1}}\right)^{3}\right\rangle=\sum_{\vec{k}=\vec{p}+\vec{q}} k_{1} p_{1} q_{1} a_{1}(\vec{k}) a_{1}(\vec{p}) a_{1}(\vec{q}) \sin \left[\phi_{1}(\vec{p})+\phi_{1}(\vec{q})-\phi_{1}(\vec{k})\right] .
$$

Here $a_{1}$ is the amplitude, and $\phi_{1}$ is the phase. In particular, a triad phase, $\phi_{1}(\vec{p})+\phi_{1}(\vec{q})-\phi_{1}(\vec{k})$, is explicitly represented on the right-hand side, indicating the link between $S_{k}$ and the phase of velocities (see Refs. [11,38-40] for more discussions). This expression explicitly illustrates that 


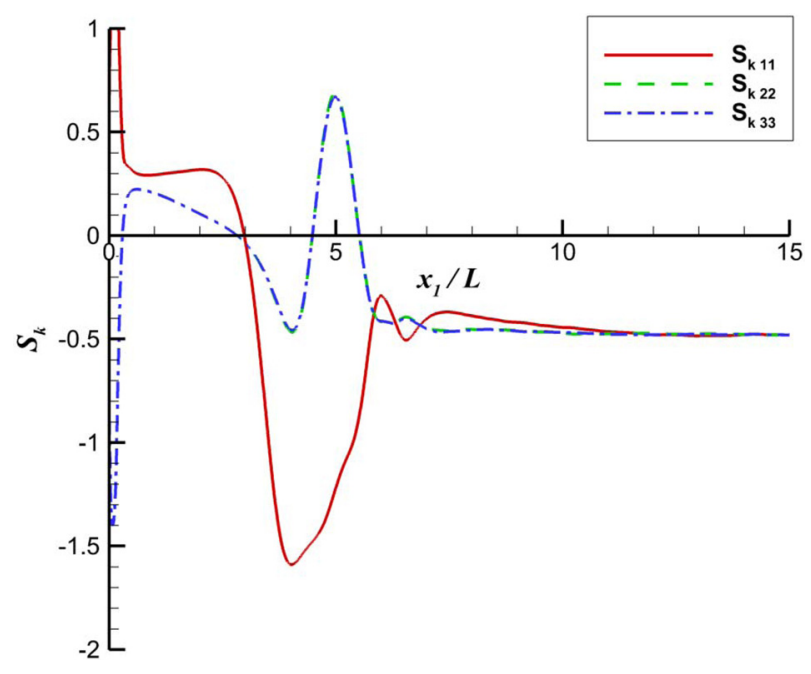

FIG. 17. Evolution of the the skewness values of $\partial u_{1} / \partial x_{1}, \partial u_{2} / \partial x_{2}$, and $\partial u_{3} / \partial x_{3}$ in the two-scale wake, respectively.

both the amplitudes and the phases can affect the evolution of $S_{k}$. Considering the coupling of the phase evolution and amplitude, the related analysis will be much more complicated and is expected to be investigated in the future.

In addition, we remark that the present evidence and analysis are still not sufficient to assert that the skewness is positive in all situations, because laminar-turbulent transition could be triggered by more types of boundary conditions and initial perturbations. However, the value of our finding is to stimulate further observations of $S_{k}$ in future transition studies in order to confirm the universality of the emergence of positive $S_{k}$. Since the positive $S_{k}$ is a direct consequence of the nonlinear interaction of different Fourier modes, which is considered as the early fingerprint of transition, a new criterion for determining the transition onset may be constructed by observation of $S_{k}$, which is superior to the skin-friction coefficient $c_{f}$ as in many engineering applications because the former is easier to be measured experimentally.

Finally, we comment that although the present study considers only the skewness of the longitudinal component of velocity gradient in the streamwise direction, the anisotropy of the sixth-order velocity gradient tensor, $\left\langle\frac{\partial u_{i}}{\partial u_{j}} \frac{\partial u_{k}}{\partial u_{l}} \frac{\partial u_{m}}{\partial u_{n}}\right\rangle$, is an interesting topic [41]. For example, in the two-scale wake case, we can calculate more longitudinal components and show the values of the skewness of $\partial u_{1} / \partial x_{1}, \partial u_{2} / \partial x_{2}$, and $\partial u_{3} / \partial x_{3}$ (denoted as $S_{k 11}, S_{k 22}$, and $S_{k 33}$, respectively) in Fig. 17. The curves of $S_{k 22}$ and $S_{k 33}$ coincide because of symmetry. Although the values of $S_{k 22}$ and $S_{k 33}$ are not equal to $S_{k 11}$, it is interesting that they have some qualitative similarities: (1) In the region of $x_{1} / L>12$ the values of skewness coincide, because there is no large-scale shear structure and the flow is quasi-isotropic. We remark that even in anisotropic flows, the skewness of streamwise velocity gradient might also be approximately used together with the Karman-Howarth equation of isotropic turbulence. For example, in Ref. [42] the subgrid-scale model is derived with local isotropy assumption, but the longitudinal direction is specifically selected as the streamwise direction in channel flow (see Fig. 12 of Ref. [42]). This indicates that the skewness of streamwise velocity gradient is, at least, a characteristic quantity in anisotropic flows. (2) Similar to $S_{k 11}$, the values of $S_{k 22}$ and $S_{k 33}$ are positive in most of the early transition region $\left(0.05<x_{1} / L<3\right)$, although the exact values are not the same. We then expect that these interesting facts can inspire future studies on the anisotropy of the sixth-order velocity gradient tensor in anisotropic flows. 


\section{ACKNOWLEDGMENTS}

We are grateful to J. Fang for providing the DNS code of the two-scale wake, and to H. Xu and W. Bos for helpful discussions. This work is supported by the National Natural Science Foundation of China (Grants No. 11772032, No. 51976006, No. U20B2003, and No. 11772224).

\section{APPENDIX: DETAILS FOR OBTAINING THE SPANWISE VELOCITIES (18)}

We want to find the variable separation solutions of Eq. (15) with boundary condition (16), written as $U_{2}\left(\xi_{2}, \xi_{3}, t\right)=A\left(\xi_{2}, \xi_{3}\right) T(t)$. Moreover, we assume that $\nabla^{2} A$ can also be written in variable separation form, i.e., $\nabla^{2} A=Y\left(\xi_{2}\right) Z\left(\xi_{3}\right)$.

Solving these equations by using the variable separation method, we obtain the following nontrivial solutions corresponding to a temporal evolution rate $T(t)=\exp (-\lambda t / \operatorname{Re})$ :

$$
\begin{aligned}
A\left(\xi_{2}, \xi_{3}\right) & =\sum_{i} C_{1 i} \sin \left(2 k_{1 i} \pi \frac{\xi_{2}}{L}\right) \cosh \left(2 k_{1 i} \pi \frac{\xi_{3}}{L}\right) \\
& +\sum_{i} C_{2 i} \sin \left(2 k_{2 i} \pi \frac{\xi_{2}}{L}\right) \cos \left(\sqrt{\lambda-4 k_{2 i}^{2} \pi^{2} / L^{2}} \xi_{3}\right) \\
& +C_{3}\left[\frac{\sin \left(\sqrt{\lambda} \xi_{2}\right)}{\sin (\sqrt{\lambda} L / 2)}-\frac{2 \xi_{2}}{L}\right] \\
& +C_{4}\left[\frac{\sinh \left(\sqrt{\lambda} \xi_{2}\right)}{\sinh (\sqrt{\lambda} L / 2)}-\frac{2 \xi_{2}}{L}\right] \cos \left(\sqrt{\lambda} \xi_{3}\right) \\
& +\sum_{i} C_{5 i}\left[\frac{\sin \left(\sqrt{\lambda+p_{i}} \xi_{2}\right)}{\sin \left(\sqrt{\lambda+p_{i}} L / 2\right)}-\frac{\sin \left(\sqrt{p_{i}} \xi_{2}\right)}{\sin \left(\sqrt{p_{i}} L / 2\right)}\right] \cosh \left(\sqrt{p_{i}} \xi_{3}\right),
\end{aligned}
$$

with $k_{1 i}, k_{2 i} \in \mathbb{N}^{+}, C_{\bullet} \in \mathbb{R}$, and $\lambda, p_{i} \in \mathbb{R} \backslash\{0\}$. Using the continuity equation (17), we can calculate the distribution of $U_{3}$. Considering the boundary condition for $U_{3}$, a similar expression to (16), all possible solutions for $A$ are

$$
\begin{aligned}
A\left(\xi_{2}, \xi_{3}\right) & =\sum_{i} C_{2 i} \sin \left(2 k_{2 i} \pi \frac{\xi_{2}}{L}\right) \cos \left(\sqrt{\lambda-4 k_{2 i}^{2} \pi^{2} / L^{2}} \xi_{3}\right) \\
& +C_{4}\left[\frac{\sinh \left(\sqrt{\lambda} \xi_{2}\right)}{\sinh (\sqrt{\lambda} L / 2)}-\frac{2 \xi_{2}}{L}\right] \cos \left(\sqrt{\lambda} \xi_{3}\right) \\
& +\sum_{i} C_{5 i}\left[\frac{\sin \left(\sqrt{\lambda+p_{i}} \xi_{2}\right)}{\sin \left(\sqrt{\lambda+p_{i}} L / 2\right)}-\frac{\sin \left(\sqrt{p_{i}} \xi_{2}\right)}{\sin \left(\sqrt{p_{i}} L / 2\right)}\right] \cosh \left(\sqrt{p_{i}} \xi_{3}\right)
\end{aligned}
$$

with either

$$
C_{4} \neq 0, \quad \lambda-4 k_{2 i}^{2} \pi^{2} / L^{2}=4 n_{1 i}^{2} \pi^{2} / L^{2}, \quad \lambda=4 n_{2}^{2} \pi^{2} / L^{2}, \quad p_{i}=-4 n_{3 i}^{2} \pi^{2} / L^{2}, \quad \forall i
$$

or

$$
C_{4}=0, \quad \lambda-4 k_{2 i}^{2} \pi^{2} / L^{2}=4 n_{1 i}^{2} \pi^{2} / L^{2}, \quad p_{i}=-4 n_{3 i}^{2} \pi^{2} / L^{2}, \quad \forall i,
$$

where $n_{1 i}, n_{2}, n_{3 i} \in \mathbb{N}^{+}$. Following Waleffe [30], we further assume that the temporal evolution is relatively weak, such that the flow can be written as a locally steady form. From assumption (5) we focus on the two largest scales, and thus we take the Fourier transform and perform large-scale truncation on these possible solutions. All possible solutions for $U_{2}$ are therefore

$$
U_{2}\left(\xi_{2}, \xi_{3}\right)=C_{21} \sin \left(2 \pi \xi_{2} / L\right) \cos \left(4 \pi \xi_{3} / L\right)+C_{22} \sin \left(4 \pi \xi_{2} / L\right) \cos \left(2 \pi \xi_{3} / L\right),
$$


which corresponds to $k_{21}=1, k_{22}=2$, and $\lambda=20 \pi^{2} / L^{2}$ in Eq. (A2).

We further use the continuity equation (17) to calculate $U_{3}$, and compare the expression between $U_{2}$ and $U_{3}$, respectively. Due to assumption (2), the expressions of $U_{2}$ and $U_{3}$ should be symmetric, leading to the only final expression (18).

[1] A. N. Kolmogorov, The local structure of turbulence in incompressible viscous fluid for very large Reynolds number, Proc. Math. Phys. Sci. 30, 301 (1941).

[2] W. J. T. Bos, L. Chevillard, J. F. Scott, and R. Rubinstein, Reynolds number effect on the velocity increment skewness in isotropic turbulence, Phys. Fluids 24, 015108 (2012).

[3] G. K. Batchelor, The Theory of Homogeneous Turbulence (Cambridge University Press, Cambridge, 1953).

[4] C. W. Van Atta and R. A. Antonia, Reynolds number dependence of skewness and flatness factors of turbulent velocity derivatives, Phys. Fluids 23, 252 (1980).

[5] F. Thiesset, R. A. Antonia, and L. Djenidi, Consequences of self-preservation on the axis of a turbulent round jet, J. Fluid Mech. 748, R2 (2014).

[6] S. L. Tang, R. A. Antonia, L. Djenidi, and Y. Zhou, Transport equation for the isotropic turbulent energy dissipation rate in the far-wake of a circular cylinder, J. Fluid Mech. 784, 109 (2015).

[7] L. Djenidi and R. A. Antonia, Modelling the third-order velocity structure function in the scaling range at finite Reynolds numbers, in 22nd Australasian Fluid Mechanics Conference AFMC2020, 6-10 December (Brisbane, Australia, 2020).

[8] R. H. Kraichnan, Inertial ranges in two-dimensional turbulence, Phys. Fluids 10, 1417 (1967).

[9] F. Liu, L. P. Lu, and L. Fang, Non-equilibrium turbulent phenomena in transitional channel flows, J. Turbul. 19, 731 (2018).

[10] L. Fang, T. Wu, and W. J. T. Bos, Staircase scaling of short-time energy transfer in turbulence, J. Turbul. 21, 234 (2020).

[11] J. Chai, T. Wu, and L. Fang, Single-scale two-dimensional-three-component generalized-Beltrami-flow solutions of incompressible Navier-Stokes equations, Phys. Lett. A 384, 126857 (2020).

[12] L. Fang, W. J. T. Bos., L. Shao, and J.-P. Bertoglio, Time-reversibility of Navier-Stokes turbulence and its implication for subgrid scale models, J. Turbul. 13, N3 (2012).

[13] L. Fang, Y. Zhu, Y. W. Liu, and L. P. Lu, Spectral non-equilibrium property in homogeneous isotropic turbulence and its implication in subgrid-scale modeling, Phys. Lett. A 379, 2331 (2015).

[14] F. Liu, L. P. Lu, W. J. T. Bos, and L. Fang, Assessing the nonequilibrium of decaying turbulence with reversed initial fields, Phys. Rev. Fluids 4, 084603 (2019).

[15] R. J. Hearst and P. Lavoie, Velocity derivative skewness in fractal-generated, non-equilibrium grid turbulence, Phys. Fluids 27, 071701 (2015).

[16] J. C. Isaza, R. Salazar, and Z. Warhaft, On grid-generated turbulence in the near- and far field regions, J. Fluid Mech. 753, 402 (2014).

[17] L. Fang, H. K. Zhao, L. P. Lu, Y. W. Liu, and H. Yan, Quantitative description of non-equilibrium turbulent phenomena in compressors, Aerosp. Sci. Technol. 71, 78 (2017).

[18] J. W. Kim and D. J. Lee, Generalized characteristic boundary conditions for computational aeroacoustics, part 2, AIAA J. 42, 47 (2004).

[19] J. D. Anderson, Fundamentals of Aerodynamics, 4th ed. (McGraw-Hill, New York, 2007).

[20] J. Fang, Y. F. Yao, Z. R. Li, and L. P. Lu, Investigation of low-dissipation monotonicity-preserving scheme for direct numerical simulation of compressible turbulent flows, Comput. Fluids 104, 55 (2014).

[21] J. Fang, Y. F. Yao, A. A. Zheltovodov, Z. R. Li, and L. P. Lu, Direct numerical simulation of supersonic turbulent flows around a tandem expansion-compression corner, Phys. Fluids 27, 125104 (2015).

[22] S. K. Lele, Compact finite difference schemes with spectral-like resolution, J. Comput. Phys. 103, 16 (1992). 
[23] S. Gottlieb and C.-W. Shu, Total variation diminishing Runge-Kutta schemes, Math. Comput. 67, 73 (1998).

[24] N. A. Adams and K. Shariff, A high-resolution hybrid compact-ENO scheme for shock-turbulence interaction problems, J. Comput. Phys. 127, 27 (1996).

[25] S. Laizet, J. Nedić, and J. C. Vassilicos, Influence of the spatial resolution on fine-scale features in DNS of turbulence generated by a single square grid, Int. J. Comut. Fluid Dyn. 29, 286 (2015).

[26] Y. Zhou and J. C. Vassilicos, Related self-similar statistics of the turbulent/non-turbulent interface and the turbulence dissipation, J. Fluid Mech. 821, 440 (2017).

[27] S. C. Tao and Y. Zhou, Turbulent flows around side-by-side cylinders with regular and multiscale arrangements, Phys. Rev. Fluids 4, 124602 (2019).

[28] S. Laizet, J. C. Vassilicos, and C. Cambon, Interscale energy transfer in decaying turbulence and vorticitystrain-rate dynamics in grid-generated turbulence, Fluid Dyn. Res. 45, 061408 (2013).

[29] Y. Zhou, K. Nagata, Y. Sakai, H. Suzuki, Y. Ito, O. Terashima, and T. Hayase, Relevance of turbulence behind the single square grid to turbulence generated by regular- and multiscale-grids, Phys. Fluids 26, 075105 (2014).

[30] F. Waleffe, On a self-sustaining process in shear flows, Phys. Fluids 9, 883 (1997).

[31] A. Zhang, M. Dong, and Y. Zhang, Receptivity of secondary instability modes in streaky boundary layers, Phys. Fluids 30, 114102 (2018).

[32] S. L. Tang, R. A. Antonia, L. Djenidi, H. Abe, T. Zhou, L. Danaila, and Y. Zhou, Transport equation for the mean turbulent energy dissipation rate on the centreline of a fully developed channel flow, J. Fluid Mech. 777, 151 (2015).

[33] R. A. Antonia, S. L. Tang, L. Djenidi, and L. Danaila, Boundedness of the velocity derivative skewness in various turbulent flows, J. Fluid Mech. 781, 727 (2015).

[34] J. Wyngaard and S. Clifford, Taylor's hypothesis and high-frequency turbulence spectra, J. Atmos. Sci. 34, 922 (1977).

[35] Z. F. Fan and M. Dong, Multiple eigenmodes of the Rayleigh-Taylor instability observed for a fluid interface with smoothly varying density. III. Excitation and nonlinear evolution, Phys. Rev. E 101, 063103 (2020).

[36] C. X. Yu, C. Xue, J. Liu, X. Y. Hu, Y. Y. Liu, W. H. Ye, L. F. Wang, J. F. Wu, and Z. F. Fan, Multiple eigenmodes of the Rayleigh-Taylor instability observed for a fluid interface with smoothly varying density, Phys. Rev. E 97, 013102 (2018).

[37] M. Dong, Z. F. Fan, and C. X. Yu, Multiple eigenmodes of the Rayleigh-Taylor instability observed for a fluid interface with smoothly varying density. II. Asymptotic solution and its interpretation, Phys. Rev. E 99, 013109 (2019).

[38] M. Buzzicotti, B. P. Murray, L. Biferale, and M. D. Bustamante, Phase and precession evolution in the Burgers equation, Eur. Phys. J. E 39, 34 (2016).

[39] B. P. Murray and M. D. Bustamante, Energy flux enhancement, intermittency and turbulence via fourier triad phase dynamics in the 1-D Burgers equation, J. Fluid Mech. 850, 624 (2018).

[40] T. Wu, L. Fang, and Z. Wang, Exact time scale of energy exchange in triad interactions of homogeneous isotropic turbulence, Phys. Fluids 33, 035136 (2021).

[41] F. H. Champagne, The fine-scale structure of the turbulent velocity field, J. Fluid Mech. 86, 67 (1978).

[42] G. X. Cui, H. B. Zhou, Z. S. Zhang, and L. Shao, A new dynamic subgrid eddy viscosity model with application to turbulent channel flow, Phys. Fluids 16, 2835 (2004). 